Sādhanā Vol. 40, Part 6, September 2015, pp. 1769-1792. (C) Indian Academy of Sciences

\title{
Attribute association based privacy preservation for multi trust level environment
}

\author{
R PRAVEENA PRIYADARSINI ${ }^{1, *}$, M L VALARMATHI $^{2}$ and \\ S SIVAKUMARI ${ }^{1}$
}

${ }^{1}$ Dept of Computer Science and Engineering, Faculty of Engineering, Avinashilingam Institute for Homescience and Higher Education for Women University, Coimbatore 641108, India

${ }^{2}$ Dept of Computer Science and Engineering, Government College of Technology, Coimbatore 641025 , India

e-mail: praveena.priya04@gmail.com

MS received 29 August 2014; revised 16 March 2015; accepted 6 June 2015

\begin{abstract}
Enormous amount of e-data is collected world-wide by organizations for the purpose of their research and decision making. The availability of this heterogeneous, sensitive information in e-databases poses a threat to the privacy of the individual or organization on which the data is collected. Privacy Preserving Data Mining [PPDM] is a field of research which concentrates on preserving data privacy during the process of data mining. This paper proposes a two level partition and perturbation frame work to release multiple copies of privacy preserved datasets in Multi Trust Level [MTL] scenario that can prevent linking and diversity attack. The framework proposes two methods namely, Entropy based Attribute Privacy Preservation [EAPP] and Information Gain based Attribute Privacy Preservation [IGAPP] for privacy preservation in MTL environment. The two methods perform vertical and horizontal partitioning of data for privacy preservation. Simple K-Means clustering algorithm with cluster size 2 using both Euclidean and Manhattan distance functions are used for horizontal partitioning. The vertical partitioning of attributes within the cluster is performed based on their entropy value that indicates its one way association with its class in EAPP method and Information Gain [IG] value of the attributes that indicates the two way associations with class in IGAPP method. The attributes in the clusters are subjected to privacy preservation technique based on their entropy and IG values in EAPP and IGAPP methods, respectively. The effect of distance in clustering the data points on privacy preservation and the ability of the privacy preserved datasets generated using the proposed methods to prevent privacy attacks are studied using variance, rank distortion and utility metrics. Real life medical and bench mark adult data sets have been used here for experimentation. The results show that the generated datasets exhibit good variance and rank distortion values and hence can
\end{abstract}

${ }^{*}$ For correspondence 
prevent diversity and linking attacks in MTL environment. Also, the privacy preserved datasets have comparable utility on selected classification and clustering algorithms with original and L-Diversified datasets.

Keywords. Privacy preserving data mining; multi trust level environment; entropy, information gain; diversity and linking attacks; attribute association.

\section{Introduction}

Data are collected both online and offline for various purpose by organizations. These data are used by data brokers to extract sensitive knowledge about data owners which compromises their privacy. Thus, protecting the privacy of the individual or organization during data collection and publication has become a vital research issue. Privacy Preserving Data Mining [PPDM] is a field of research that strives to protect the privacy of data during the process of data mining. Some of the techniques used for preserving privacy in PPDM are anonymization, randomization, and perturbation, distributed privacy preservation (Charu Aggarwal \& Philip Yu 2008). The privacy preservation techniques used for data masking usually assumes a single trust level scenario on data miners. Yaping le et al (2012) have introduced a scenario in PPDM where the data owner trust the data miner at various levels and privacy preservation is applied on the published data sets based on the trust levels of the miner. Figure 1 shows the multi trust level scenario of privacy preserving data mining.

In multi-trust level scenario, the datasets are distributed to recipients based on their trust level. The main challenge of MTL environment is to produce versions of privacy preserved datasets

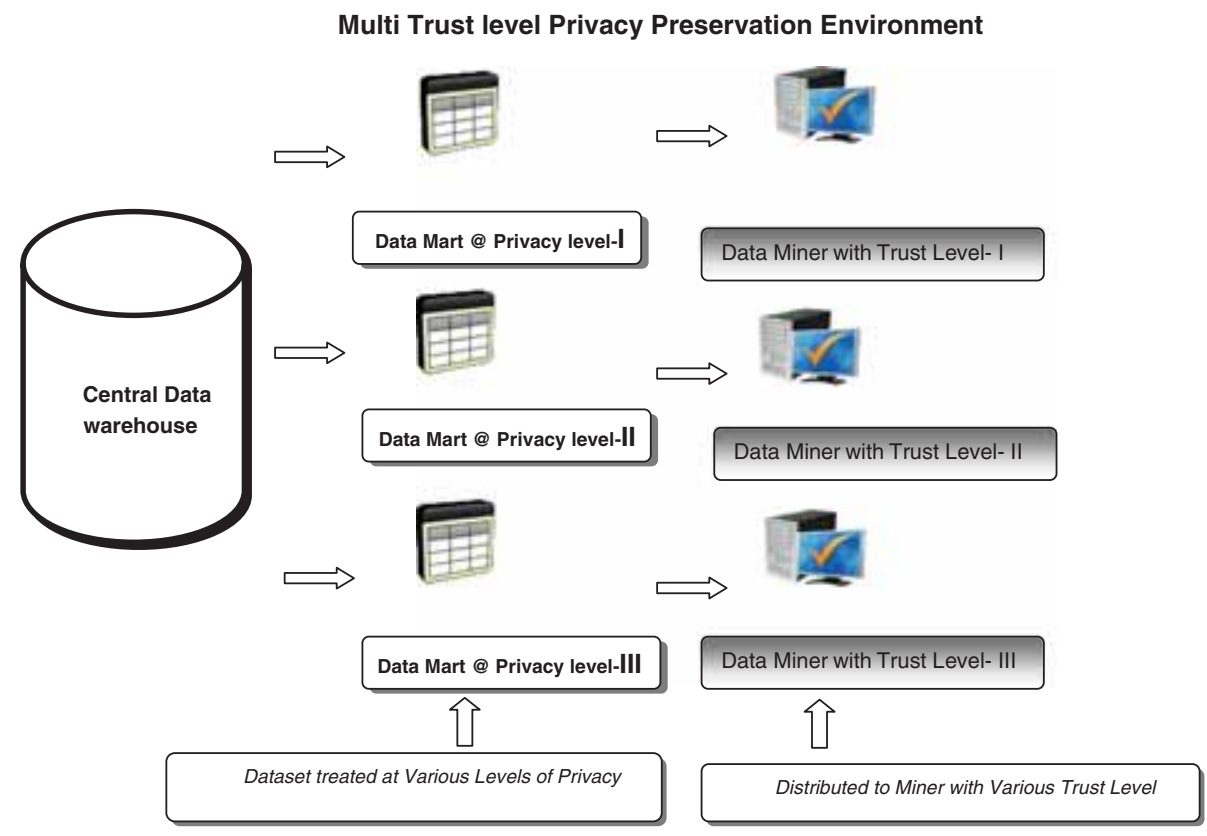

Figure 1. Multi-trust level privacy preservation scenario. 
with good variance such that it can counter diversity and linking attacks. The main aim of this work is to address the above challenge by using two level partitioning and perturbation framework. The proposed framework aims to study the effect of horizontal and vertical distance between the data points and attributes respectively during the process of privacy preservation.

At level one, horizontal partition is performed. As Charu Aggarwal (2008) suggested, closer the distance between the data points the lesser is the loss of information due to privacy preservation. Hence, the data points are clustered based on Eculidian and Manhattan distance using $\mathrm{K}$-means clustering algorithm and the effect of these distances on privacy preservation is studied.

At level two, vertical partitioning is performed based on association between the attributes with its class and with other attribute using Information theoretic measures and symmetry axioms. These associations between attributes are used for identifying the sensitivity of the attributes automatically. Attributes are then subjected to privacy preservation based on sensitivity. This automatic identification of sensitivity helps in segregation of attributes as quasi identifiers and sensitive attributes which in-turn helps in solving the problem of attribute segregation in high dimensional datasets and data streams.

Thus, the proposed framework has two-dimensional privacy preservation methods EAPP and IGAPP for privacy preservation. These proposed methods use slicing technique (Tiancheng Li et al 2012) that partitions the data two-dimensionally, both vertical and horizontal. In the proposed methods horizontal partitioning of data is performed using $\mathrm{k}$ - means clustering algorithm using Euclidean and Manhattan distance. The vertical partitioning of attributes within the cluster is performed by using entropy values of the attributes in EAPP method and IG value of the attributes within the cluster in IGAPP method. Privacy preservation techniques are applied to the attributes based on these values.

The class labels are attached to the data within the cluster. Based on the distance functions, the proposed methods EAPP and IGAPP will produce two versions of privacy preserved datasets each. The datasets used in this work are real life medical data set and adult dataset obtained from UCI machine learning repository (http://archive.ics.uci.edu). The effect of Manhattan and Euclidean distance on privacy preservation of data is studied. The ability to counter the attacks like linking and diversity have been studied using variance and distortion metrics on privacy preserved datasets. The utility of the privacy preserved datasets obtained using the proposed methods are evaluated by applying the utility metrics on selected algorithms of classification and clustering.

The paper is organized as follows: Section 2 describes the literature survey, section 3 explains the proposed methodology, section 4 describes the horizontal and vertical partitioning for privacy preservation of the datasets, section 5 gives the dataset descriptions, section 6 describes the evaluation metrics use in this work, section 7 explains the experimental result and discussions and section 8 gives the conclusions.

\section{Literature survey}

Privacy preservation during data analysis has two settings. The first one is interactive setting where the database that is in the custody of the trusted server. This server takes care of the privacy issues. The second setting is the non-interactive setting where the data custodian releases the privacy preserved version of the raw data (Danker \& El Emam 2012).

Fung et al (2009) proposed a framework which has two dimension, one it preserves the individual privacy and secondly, it also protects and keeps the usefulness of the information during 
cluster analysis. The class labels have been preserved while masking the relative data of the tuple.

Dinusha Vatsalan et al (2013) discussed about the issues in privacy preservation when the databases are allowed to be linked from various data bases across the organization. This issue is commonly called as Privacy Preserving Record Linking (PPRL). Dan Zhu et al (2009) used aggregation and swapping as the first step of anonymization. The best subset of tuples selected using genetic algorithm and $\mathrm{k}$ - anonymization is applied on them. Sarowar Sattar et al (2013) integrated sampling data selection technique with generalization for preserving the privacy of the published data. Chris R Giannela et al (2013) proposed an attack known as known input attack. Using real and synthetic data, the work has proved that with significantly small number of data tuples which is less than the dimension of the dataset, privacy can be breached in high probability.

Srujana Marugu \& Joydeep Ghosh (2005) proposed a frame work for distributed clustering where each data owner applies customized privacy on their data and this version is stored in the centralized server. Josep Domingo - Ferrar (2008) discussed about the methods used for Statistical Disclosure Control (SDC) of sensitive data like e-commerce, health and official statistical data.

Ming Hau \& Jian Pei (2008) have introduced utility based anonymization, top down specialization and progressive discloser algorithm for privacy preservation in classification problems. Murat Kantanlioglu (2008) discussed about the privacy concerns in horizontally partitioned data. The two adversarial models in Secure Multi party Computation (SMC) with semi honest and malicious adversarial model have been discussed in this work.

Lin Xiaodong et al (2005) proved that clustering based on expectation maximization limits the disclosure of data between sites in a distributed environment. The scenario called Multi-level Trust to perturbation based privacy preserving data mining where the most trusted user is given a less perturbed data and the least trusted user is given the most perturbed version of the data was introduced by (Yaping li \& Minghua Chen 2012). The diversity attack that may happen in this scenario is prevented by generating perturbed copies of dataset based on arbitrary trust level on demand.

Javier Herranz et al (2010) proposed and analysed specific privacy protection methods based on their utility and privacy preservation capacity. The statistical disclosure control [SDC] protection measures like additive and multiplicative noise addition, rank swapping, micro aggregation and IPSO methods have been used on Census and Vehicle data set. It is proved that the utility of the SDC protection methods are very close to the original dataset for classification on decision tree, naive Bayes, KNN and SVM algorithms.

Erez shmueli et al (2012) presented a top-down algorithm for anonymizing sequential releases of data. The algorithms anonymise the data using the cell generalization model that has good utility and offers privacy during kernel match joins.

Gagan Aggarwal et al (2006) proposed a new method of anonymization where cluster center value of the quasi identifiers are published instead of the original values. Shlome Berkovsky et al (2012) proposed a system where the collaborative filtering systems user profiles are distributed across multiple repositories instead of centralized site to preserve their privacy. The obfuscation is performed on the user profile data to partially hide the actual user ratings. The quantification of randomization privacy preservation method for collaborative filtering systems is performed using Average Absolute Error metric (Songjie Gong 2011).

Md Zahidul Islam \& Lyilyana Brankovic (2011) proposed a novel technique for clustering categorical values and used it for noise addition. The proposed framework preserves the original patterns in the perturbed dataset and the correlation among the attributes.

Stanley olivera \& Osmar Zainne (2007) have designed a solution that meets the privacy requirements and also gives good utility while performing data clustering function. Weiwei Ni 
\& Zhihong Chong (2012) proposed privacy preservation methods that preserve sensitive information before publishing. In the proposed AENDO method, private data are protected by either swapping the values or by substitution before releasing it to the public based on the data distributions. Tiancheng Li et al (2012) presented a technique called slicing which horizontally partitions the data and applies perturbation vertically. Slicing gives good data utility that can prevent membership disclosure and can also handle high-dimensional data.

Weijia Yang \& Shang Tang Haung (2008) used clustering method for privacy preservation in multi-party computing environment. This method allows customized privacy preservation by the owners to their desired level. Customized two phase perturbation where the data provider sets the privacy level according to their choice and yields good datamining utility was proposed by Li Liu et al (2008). Rashid Hussain Khokhar et al (2014) proposed a privacy preservation model that gives a analytical cost model that helps the health information custodians to take decision while sharing personal and health specific data with others. Gal et al (2014) proposed a framework where de-identification of data is customized based on the specific needs of the data recipient. Grigorios Loukides et al (2014) used a method which prevents linkage attacks among medical records using disassociation method. Chunks of similar records are created using $\mathrm{K}^{\mathrm{m}}$ anonymization method for de-identification of records. Wu \& Zhang (2004) used feature selection for classifying high dimensional data set. They have proposed balanced information gain for measuring the contribution of each feature for classification function. Their work is based on the assumption that mutual information is termed as information gain in machine learning. Information theory based metric is useful in identifying dependency between attributes (Edoardo M Airoldi et al 2011). Evfimevski et al (2003) presented amplification approach to privacy that uses randomization method that can limit several privacy breaches.

\section{Proposed methodology}

Li \& Chen (2012) proposed privacy preservation technique in multi-trust environment where noise addition based privacy preservation is performed to obtain parallel and sequential release of data versions. Likewise, Yang \& Huang (2008) allowed users to customize the privacy for each attribute in MTL scenario. But, both the work do not take into consideration the problem of finding associations between attributes and classifying them as sensitive and quasi identifiers in dataset. This work proposes a two level partitioning and perturbation method that automatically identifies the sensitivity of the attributes based on information theoretic measures and symmetric axioms such that attribute segregation and privacy preservation becomes easier in dataset. This automatic identification can solve the problem of attribute segregation in dynamic datasets.

The proposed framework contains two privacy preservation methods EAPP and IGAPP. In both the methods, partitioning of data is done in two-dimensions horizontally and vertically. After partitioning the data horizontally with K-Means clustering algorithm using either Euclidean/Manhattan distance, the clustered data are assigned to their respective class labels within the cluster.

The information content of the attributes inside the cluster is measured using entropy value in EAPP method and Information gain measure in IGAPP method. The attribute partition is done vertically based on these values. The Entropy values of attributes measure the vertical distribution of information and the one way association it has with the respective class. The Information gain value of the attributes gives the relative information of the attributes and the two ways or symmetric association with its class. 
The high frequency attributes with respect to class will have high entropy and uniform distribution. The low frequency attributes will have low entropy and uneven distribution of values. The high information gain value attributes with respect to class attribute will give more information about the class attribute. Thus, by grouping the attributes on these values and applying privacy preservation techniques on these attributes at various levels, the sensitive and private information contained in them can be preserved for privacy attacks.

Hui Wang \& Ruilin Liu (2011) proved that cross attribute correlation due to functional dependency may lead to potential privacy threat in a dataset. Entropy and information gain are symmetrical measures. These measures are a desirable property for an inter-correlation between attribute (Hall M 1999). As a symmetric measure, it is assumed that perturbation based on Entropy and Information gain values of an attribute can prevent privacy due to cross correlation among attributes and linking attacks. In the proposed work, vertical partitioning of attributes using Entropy or IG values higher than the mean entropy or IG value of the attributes within the cluster are considered as highly dependent attribute on class and is perturbed using generalization privacy preservation technique.

The attributes with Entropy or IG values lower than the mean entropy or IG value of the attributes within the cluster are considered to be less frequent or less dependent on the class attribute. Hence, data swapping perturbation technique is applied on them. The privacy preserved dataset

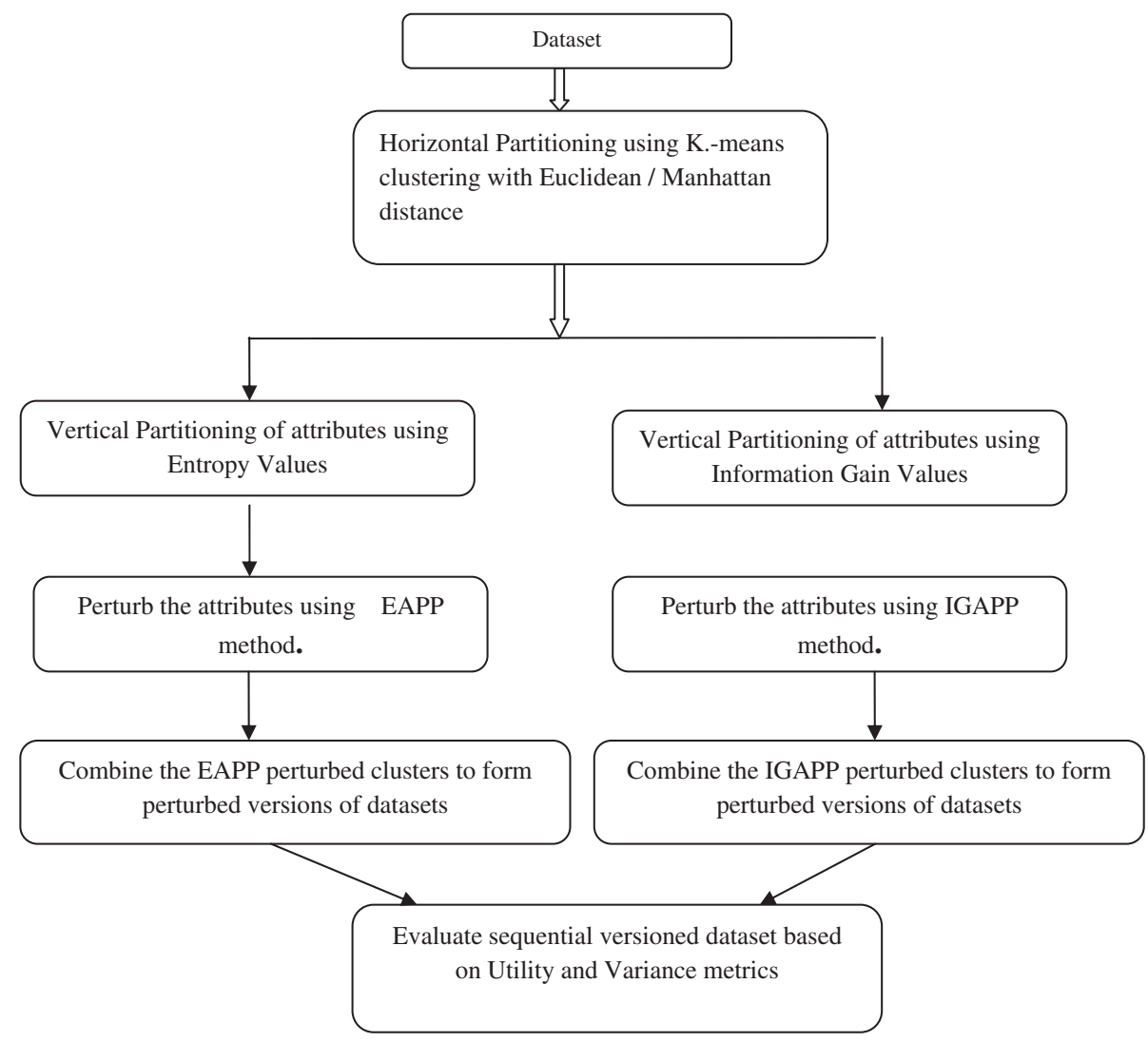

Figure 2. Proposed methodology. 
produced by applying the proposed methods will have an attributes that may be subjected to two different privacy preservation techniques. Figure 2 shows the proposed methodology and framework for vertical partitioning and privacy preservation.

\section{Horizontal partitioning of dataset using K-means clustering}

Charu Aggarwal (2008) discussed that maximum Euclidean distance between data points in a group than larger is the loss of information. Thus, to limit the loss of information during privacy preservation, in the proposed work attributes in the datasets are horizontally clustered based on both Euclidean and Manhattan distance using K-means clustering. This algorithm is a simple and effective clustering algorithm where the final clusters are sensitive to the initial cluster centers. The K-Means algorithm partitions the data such that each cluster center is represented by their mean value of the object in the cluster (Jiawei Han et al 2011).

\subsection{Proposed vertical partitioning and privacy preservation of dataset}

The attributes in the clusters are partitioned vertically into two groups based on their information as low and high in IGAPP method. The same procedure is extended using entropy as low and high attributes in EAPP method.

4.1a Perturbation techniques: The two perturbation techniques used in the proposed algorithms are Data swapping and Generalization

4.1b Data swapping privacy preservation technique: This technique is used in both the proposed methods, EAPP and IGAPP for vertical perturbation of data. Data swapping is related

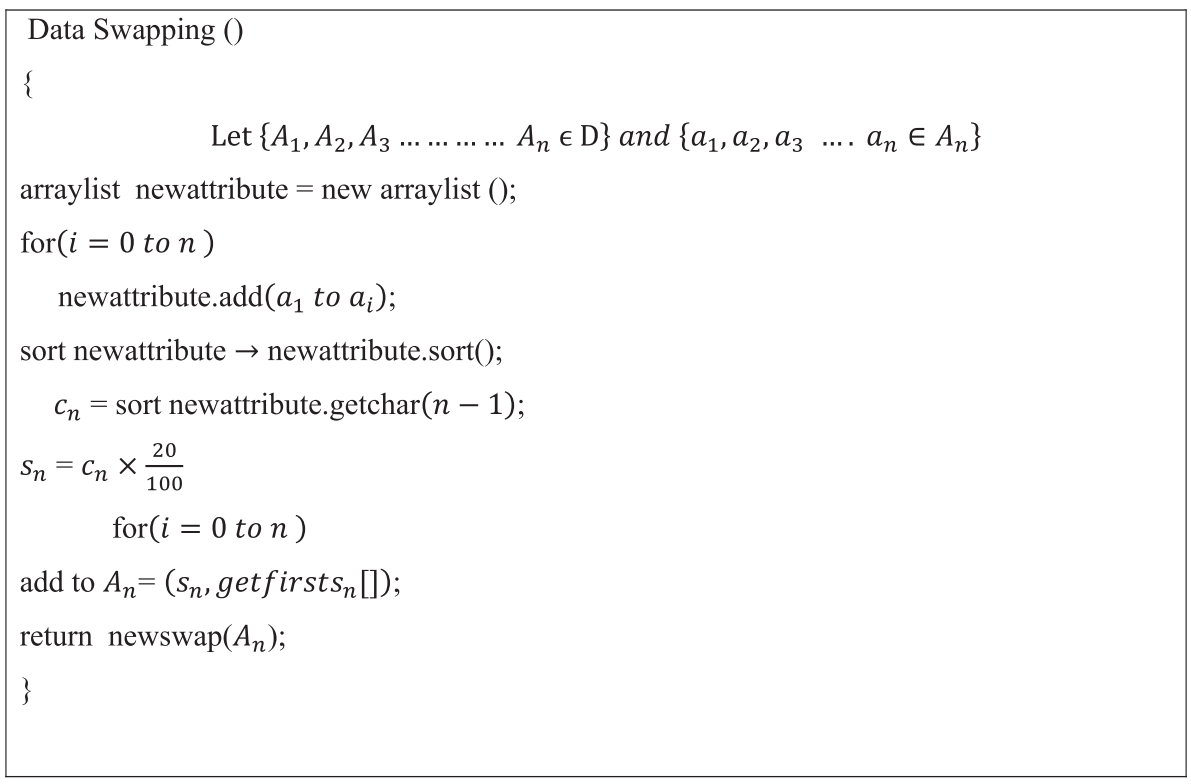

Figure 3. Data swapping algorithm. 


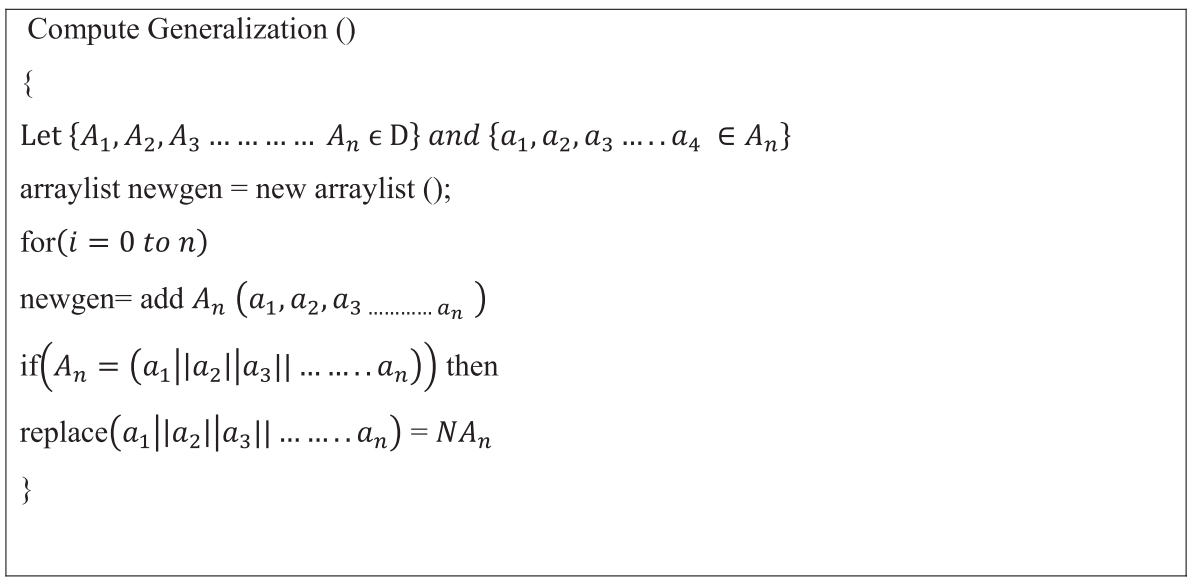

Figure 4. Generalization algorithm.

to randomization privacy preservation technique. This allows the values of the attribute to be swapped across different records such that the privacy of the data is preserved. Effect of perturbation can be increased or decreased by varying the percentage of values to be swapped. This method has an advantage of computing aggregation of attributes without compromising on their privacy (Aggarwal \& Philip 2008). The algorithm for the same is given in figure 3:

4.1c Generalization privacy preservation technique: Generalization is a privacy preservation technique, where for a categorical attribute, a specific value is replaced by a general value and for a numeric attribute, no predetermined taxonomy is suggested. Generalization of data will produce a less precise version of the data (Sweeney 2002; Samarati 2001). The algorithm for the same is given in figure 4 .

\subsection{Entropy based attribute privacy preservation [EAPP]}

In EAPP method the following assumption is made:

Assumption 1: It is assumed that attributes $\mathrm{a}_{1}, \mathrm{a}_{2} \ldots \ldots \ldots \ldots \mathrm{a}_{\mathrm{n}} \in$ Dataset $\mathrm{D}$ and $\mathrm{a}_{\mathrm{x} 1}, \mathrm{a}_{\mathrm{x} 2} \ldots \mathrm{a}_{\mathrm{xn}} \subseteq \mathrm{D}$ are the attributes that have higher entropy value than the mean entropy value of the attributes within the cluster and hence they are uniformly distributed attributes. The greater the entropy value of the attribute with class, the more uniform distribution of data with respect to class and hence they are assumed to be more associative and are subjected to high level of privacy preservation. Let $\mathrm{a}_{\mathrm{y} 1}, \mathrm{a}_{\mathrm{y} 2} \ldots \mathrm{a}_{\mathrm{yn}} \subseteq \mathrm{D}$ be the attributes that have lower entropy values than the mean entropy value of the attributes within the cluster and hence they are unevenly distributed attributes. The lower the entropy value, the lesser the uniformity and it is assumed that the data carries and low level of association with class and hence it is subjected to a lesser level of privacy preservation.

In EAPP method, the distribution or concentration of data values within a cluster are measured by calculating the entropy measurement of each attribute within the cluster after assigning their respective class labels. 
4.2a Entropy measure: The amount of self-information contained in a data is considered to be its entropy measure. Entropy is also considered as a generic measure of uncertainty and is applied in various clustering analysis. The entropy of an attribute is calculated using Eq. (1)

$$
\text { Entropy }(\mathrm{A})=-\sum_{\mathrm{k}=1}^{\mathrm{m}} \mathrm{P}_{\mathrm{k}} \log _{2}\left(\mathrm{P}_{\mathrm{k}}\right)
$$

$\mathrm{P}_{\mathrm{K}}$ can also be rewritten as given in Eq. (2)

$$
P_{k}=\frac{n\left(A\left(v_{i}\right)\right)}{n\left(D\left(c_{i}\right)\right)}
$$

where $\left\{A_{1}, A_{2}, A_{3} \ldots \ldots \ldots A_{n} \in D\right\}$ are the attributes that belong to dataset $\mathrm{D}$,

$\left\{c_{1}, c_{2}, c_{3} \ldots c_{n} \in c\right\}$ are the values of a class attribute $\mathrm{C}$,

$\left\{v_{1}, v_{2}, v_{3} \ldots \ldots v_{n} \in v\right\}$ are the values of an Attribute A.

$\left(n\left(D\left(c_{i}\right)\right)\right)=$ No of tuples that belong to $c_{i}$ value of a class attribute.

$\left(n\left(A\left(v_{i}\right)\right)\right)=$ No of tuples belonging to $v_{i}$ value of an attribute A.

Therefore, entropy of a dataset D can be rewritten as given in Eq. (3)

$$
E(D)=-\sum_{i=1}^{i=n} \frac{n\left(A\left(v_{i}\right)\right)}{n\left(D\left(c_{i}\right)\right)} \log _{2} \frac{n\left(A\left(v_{i}\right)\right)}{n\left(D\left(c_{i}\right)\right)} .
$$

Eq. (3) gives the entropy of attribute A with respect to Class C. The general formula for entropy calculation can also be considered as joint entropy where in Eq. (4) as X may be assumed as attribute values and $\mathrm{Y}$ may be assumed as class attribute. For attribute $\mathrm{X}$ and its class $\mathrm{Y}$, the joint entropy is calculated as given in Eq. (4)

$$
H(X, Y)=-\sum_{x \in V_{x}} \sum_{y \in V_{y}} P(x, y) \log (P(x, y) .
$$

The attributes with lower entropy value divides the dataset into few large classes and attributes with higher entropy values will divide the dataset into smaller classes. High and low entropy values may not be helpful as index in attribute selection, but as joint entropy they are helpful in finding the one way association between the attributes within the dataset. As a measure of structuredness, entropy of attribute which can be used for selection of attributes can also be used to measure the association with class attribute (Yao 2003).

For an attribute A, higher entropy value means that it is from a uniform distribution of values with respect to class attribute. Based on these values, the attributes in the dataset are divided into two groups namely, Entropy high attributes (EH) and Entropy low attributes (EL). The attributes whose entropy values are above the mean entropy value of the attributes in the dataset is grouped as EH attributes and the attributes below the mean entropy value of the attributes in the dataset is grouped as EL attributes and each group is subjected to different privacy preservation techniques. These attributes are then combined to form the cluster. These horizontal clusters are then in-turn combined to form the perturbed dataset version.

4.2b Entropy Attribute Partitioning Perturbation Algorithm: As per assumption 1, the EH attributes are considered to be more uniformly distributed and hence assumed to have high one way association with the class. Hence, they are perturbed using the generalization perturbation 
Inputs: Dataset D;

Hierarchical Partitioning using K-Means

For each Cluster $C_{i}$

$$
\left\{A_{1}, A_{2}, A_{3} \ldots A_{n} \in C_{i}\right\}, \& C_{i} \subseteq \mathrm{D}
$$

For $A=1 \ldots n$, do

Compute entropy $\left(\mathrm{A}_{\mathrm{i}}\right)=\mathrm{E}\left(\mathrm{A}_{\mathrm{n}}\right)$

$$
\text { If } \mathrm{E}\left(\mathrm{A}_{\mathrm{n}}\right) \geq \sum_{i=1}^{n} \frac{E\left(A_{n}\right)}{N} \quad, \quad \text { then }
$$

Compute Generalization $\left(\mathrm{A}_{\mathrm{n}}\right)$;

Else if $\mathrm{E}\left(\mathrm{A}_{\mathrm{n}}\right) \leq \sum_{i=1}^{n} \frac{E\left(A_{n}\right)}{N}$

Compute Data Swapping $\left(A_{n}\right)$;

End if;

Figure 5. Entropy attribute partitioning perturbation algorithm.

technique. The EL attributes are considered to have skewed distribution with lower association with class and hence they are perturbed using data swapping perturbation technique and the algorithm is given in figure 5 .

Thus, after perturbation the probability distribution of attributes in EH and EL are disturbed. Thus, it will reduce the threat of cross correlation or linking attack on privacy. The algorithm will produce two privacy preserved versions of each dataset as given below:

(i) Entropy Euclidean K-means clustered dataset with cluster size $\mathrm{C}=2$

(ii) Entropy Manhattan K-Means clustered dataset with cluster size $\mathrm{C}=2$.

\subsection{Information gain based attribute privacy preservation [IGAPP]}

In IGAPP method the following assumption is made:

Assumption 2: It is assumed that attributes $\mathrm{a}_{1}, \mathrm{a}_{2} \ldots \ldots \ldots \ldots \mathrm{a}_{\mathrm{n}} \in \mathrm{D}$ and $\mathrm{a}_{\mathrm{x} 1}, \mathrm{a}_{\mathrm{x} 2} \ldots \mathrm{a}_{\mathrm{xn}} \subseteq \mathrm{D}$ are the attributes that have higher information Gain[IG] value than the mean information gain value of the attributes within the cluster. Hence, they are assumed as mutual information high and highly symmetrical attributes to the class attributes. The greater the Information Gain value, the more information the data carries. Let $\mathrm{a}_{\mathrm{y} 1}, \mathrm{a}_{\mathrm{y} 2} \ldots \mathrm{a}_{\mathrm{yn}} \subseteq \mathrm{D}$ be the attributes that have lower information gain value than the mean information gain value of the attributes within the cluster. They are assumed as mutual information low and less symmetry attributes to class attributes. The lower the Information Gain value, the less mutual information the data carries.

In the proposed IGAPP method, the attributes are partitioned vertically into two groups based on the information gain value of the attributes within the cluster. The mutual information or 
information gain is a symmetrical measure which gives the amount of information gained about Y observing X. Thus the mutual information is calculated as given in Eq. (5) below:

$$
\text { Entropy }(y)=-\sum_{y \in Y} p(y) \log _{2}(p(y)) \text {. }
$$

Entropy of $\mathrm{Y}$ after observing $\mathrm{X}$ is given in Eq. (6)

$$
H\left(\frac{y}{X}\right)=-\sum_{x \in X} P(x)-\sum_{y \in Y} P\left(\frac{y}{x}\right) \log _{2}\left(p\left(\frac{y}{x}\right)\right)
$$

The amount by which the entropy of $\mathrm{Y}$ decreases with the addition information given by $\mathrm{X}$ is called the gain of $\mathrm{Y}$ due to $\mathrm{X}$ and termed as Information gain $\mathrm{Y}$ as calculated using the following Eqs. (7, 8 and 9)

$$
\begin{gathered}
\text { Gain }=H(Y)-H\left(\frac{Y}{X}\right) \\
=H(X)-H\left(\frac{X}{Y}\right) \\
=H(Y)+H(X)-H(X, Y)
\end{gathered}
$$

The above equations on information gain or mutual information proves that $\mathrm{X}$ says as much about Y as Y says about X (Thomas Cover et al 1991). The attributes with the highest information gain maximizes the information needed to classify the types in the dataset (D) (Jiawei Han et al 2011). When the information gain of the attribute is calculated on the class attribute it gives the amount of symmetry the attribute has over its class attribute. As per symmetry dependency axioms using transitivity rule (Baixeries Jaume 2008) as given in Eq. (10), the attributes that exhibit high symmetry with class will also exhibit symmetry among themselves.

$$
X \Rightarrow Y \text { and } Y \Rightarrow Z \text { hold, then, } X \Rightarrow Z \mid Y \text { also holds }
$$

Thus, when the two attribute show high Information Gain on class attribute, they may have high mutual information or high correlation among each other.

For example, both Education and Education number attributes that belong to Adult dataset have high IG value with respect to class i.e., high symmetry with class. Hence as per symmetry transitivity dependency they will also have dependency among them as given in Eqs. (11, 12 and 13)

$$
\begin{gathered}
\text { Education } \Rightarrow \text { Class } \\
\text { Education Number } \Rightarrow \text { Class }
\end{gathered}
$$

Then according to transitivity rule

$$
\text { Class } \Rightarrow \text { Education/Education Number }
$$

Thus, Eqs. 10 and 11 indicate that Class, Education and Education Number attributes have two way associations among them. Therefore, as per symmetric transitivity rule in Eq. (10) it is assumed that Education and Education Number will also have two way associations among them as shown in Eq. (14).

$$
\text { Education } \Rightarrow \text { Education Number }
$$

Thus, when high IG values attributes are grouped and perturbed they break the symmetry among them. The resultant privacy preserved datasets can prevent the treat of privacy due to symmetric 
Inputs: Inputs: Dataset D;

Hierarchical Partitioning - K-Means

For each Cluster $C_{i}$

$$
\left\{A_{1}, A_{2}, A_{3} \ldots A_{n} \in C_{i}\right\}, \& C_{i} \subseteq \mathrm{D}
$$

For $\mathrm{A}=1 \ldots \mathrm{n}$, do

Compute Information Gain $\left(\mathrm{A}_{\mathrm{n}}\right)=\mathrm{IG}\left(\mathrm{A}_{\mathrm{n}}\right)$

$$
\text { If IG }\left(\mathrm{A}_{\mathrm{n}}\right) \geq \sum_{i=1}^{n} \frac{I G\left(A_{n}\right)}{N} \quad \text { (mean IG value of the attributes in the dataset), }
$$

then

Compute Generalization $\left(A_{n}\right)$

Else if

$$
\begin{aligned}
\mathrm{IG}\left(\mathrm{A}_{\mathrm{n}}\right) \leq \sum_{i=1}^{n} \frac{I G\left(A_{n}\right)}{N} \\
\text { Compute Data Swapping }\left(\mathrm{A}_{\mathrm{n}}\right)
\end{aligned}
$$

End if;

Figure 6. Information gain based privacy preservation algorithm.

dependency. This measure of attribute information is used as per assumption 2 to group the attributes within the horizontal partition as two groups namely, Information Gain High (IGH) attributes and Information Gain Low (IGL) attributes. These groups are subjected to different types of privacy preservation techniques.

4.3a Information Gain Privacy Preservation Algorithm: As per assumption 2, IGH attributes are considered to be more sensitive and symmetric towards class attribute and are privacy preserved using generalization perturbation technique. The IGL attributes are considered to be less sensitive and symmetric towards the class attribute and are hence privacy preserved using data swapping perturbation technique. The algorithm for the same is given in figure 6 .

Since the highly symmetric attributes are subjected to high level of privacy preservation, cross correlation among these attributes and with class attribute is expected to decrease. Also, the symmetry of highly informative attributes with the other attributes within the IGH and IGL will also decrease due to the application of privacy preservation techniques. Thus, the treat for privacy due to symmetric dependency among attributes is prevented. The proposed frame work using both vertical and horizontal partitioned of dataset will produce two privacy preserved versions of dataset as given below.

(i) Information Gain Euclidean K-means clustered dataset with cluster size 2.

(ii) Information Gain Manhattan K-means clustered dataset with cluster size $\mathrm{C}=2$.

\section{Dataset descriptions}

Two datasets namely, real life medical dataset and adult dataset are used for experimentation in this work. The two datasets have numerical and categorical attributes. Real life medical data set 
Table 1. Medical and adult dataset description.

\begin{tabular}{lcc}
\hline DATASET & MEDICAL & ADULT \\
\hline Attribute Characteristics: & Categorical, integer & Categorical, integer \\
Number of instances: & 150 & 814 \\
Number of attributes: & 13 & 14 \\
Missing values & No & Yes \\
No. of classes & 2 & 2 \\
\hline
\end{tabular}

contains 150 records collected from patients and the aim of the dataset is to find whether the disease diagnosed is breathing or non-breathing related ailments. Adult data set is obtained from UCI machine learning repository [http://archive.ics.uci.edu], where the aim is to find whether the income of a person is less than or greater than $50 \mathrm{~K}$. The description of the datasets used in this work is given in table 1 . The real life medical dataset is pre-processed using generalization method on all the sensitive attributes like address, occupation, height, weight and diagnosis, as these attributes can directly identify the entity or the person in the dataset.

\section{Evaluation metrics}

\subsection{Variance measure}

Olivera \& Zaiane (2003) have measured the difference between the actual and the perturbed values of the attributes as variance $(X-Y)$ where $\mathrm{X}$ represents original value and $\mathrm{Y}$ is the distorted value of the attribute. As the dataset used for experimentation have categorical and continuous attributes, the difference or variance of these attributes is assumed to have the values given in Eq. (15)

$$
\operatorname{Var}(X, Y)=\left\{\begin{array}{c}
\text { if } X=Y \text { then var }=0 \\
X=Y \text { then var }=1
\end{array}\right.
$$

Therefore, the mean variance of the attribute $A_{i}$ is measured using Eq. (16)

$$
\operatorname{Variance}\left(\mathrm{A}_{1}\right)=\frac{1}{N} \sum_{i=1}^{M} X-Y
$$

where $\mathrm{m}$ is the total number values in the attribute $\mathrm{A}_{\mathrm{i}}$. This can also be called as Average Absolute Deviation (AAD) of the attribute (Pang-Ningtan et al 2006) and $\mathrm{N}$ is the number of tuples in the dataset. Hence the variance of the perturbed dataset D' or AAD of the perturbed dataset D' is calculated as given in Eq. (17).

$$
\operatorname{Variance}(D)=\frac{1}{N} \sum_{i=1}^{M} A_{i}
$$

where $\mathrm{n}$ is the total number of attributes in the dataset.

\subsection{Classification accuracy}

This is defined as the ability of the classifier to classify the given dataset and the equation for the same is given in Eq. (18).

$$
\text { Classification Accuracy }=\frac{\text { No. of tuples correctly classified }}{\text { Total no. of tuples in the dataset }} \text {. }
$$




\subsection{RMSE measurement}

The Mean squared error brings out the outliers better. The square root of mean squared error gives the root mean squared error which measures the error with the same magnitude as the data being predicted (Jiawei Han et al 2011).

$$
R M S E=\sqrt{\frac{\sum_{i=1}^{d}\left(y_{i}-y_{i}^{\prime}\right)^{2}}{d}},
$$

where,

$$
\mathbf{d}=\text { No of tuples of } \mathrm{D} \text { (Dataset) }
$$

$\mathbf{y}_{\mathbf{i}}, \mathbf{y}_{\mathbf{i}}^{\prime}=$ The response variables.

\subsection{Roc-area measurement}

The ROC-Area returns the probability or ranking for the predicted class for each tuple present in the dataset. The Roc-Area values range between 0 and 1.0.The closer the value of area to 0.5 , less is the accuracy and the value is 1.0 for perfect accuracy (Jiawei Han et al 2011).

\subsection{Purity measure}

Purity is an evaluation metric that measures the quality of clustering solution (Ienco et al (2012). To calculate purity, each cluster is assigned to the majority class of the objects in the cluster. The accuracy of this assignment is then measured by counting the number of correctly assigned objects divided by the total number of objects $n$. The formula for calculating the same is given in Eq. (20):

$$
\operatorname{Purity}(C, P)=\frac{1}{n} \sum_{j}^{i} \max \left|C_{j} \cap P_{i}\right| \text {, }
$$

where $\left\{\mathrm{C}_{1} \ldots \mathrm{C}_{\mathrm{J}}\right\}$ denotes the partition built by the clustering algorithm on objects, and $\mathrm{P}=\left\{\mathrm{P}_{1}\right.$ $\left.\ldots \mathrm{P}_{\mathrm{i}}\right\}$ denotes the partition inferred by the original classification. Where $\mathrm{j}$ and $\mathrm{i}$ are respectively the number of clusters $\mid \mathrm{Cl}$ and the number of classes $\mid \mathrm{Pl}$. The value of $\mathrm{n}$ is equal to the total number of objects.

\subsection{Change of rank of features $(C P)$}

The metric CP measures the variation in the rank of the attributes before and after distortion or perturbation. If the rank of the attribute before privacy preservation is denoted as $R A V_{i}$ and after privacy preservation is denoted as $R A V_{i}^{*}$ the difference in their rank is then measured using the metric CP as given in Eq. (21):

$$
C P=\frac{\sum_{i=1}^{m}\left|R A V_{i}-R A V_{i}^{*}\right|}{m},
$$

where $R A V_{i}$ is the rank of the average value of attribute $i$, while $R A V_{i}^{*}$ denotes its rank after the distortion (Wang et al 2006). 


\subsection{Maintenance of rank of features $(C K)$}

$C K$ is the measure that gives the percentage of the attributes that keep their ranks after the distortion (Wang et al 2006). This is calculated as given in Eqs. (22 and 23).

$$
\begin{gathered}
C K=\frac{\sum_{i=1}^{m} C K}{m} \\
C K=\left\{\begin{array}{cc}
1 & \text { if } R A V_{i}=R A V_{i}^{*} \\
0 & \text { otherwise }
\end{array}\right.
\end{gathered}
$$

\section{Experimental results and discussions}

The datasets are perturbed using both generalization and rank swapping techniques. The privacy preservation technique to which the attribute is subjected is based on its entropy value in EAPP method and information gain value in IGAPP method. The accuracy, root mean square error and ROC-area values of the privacy preserved datasets are compared on real life medical and benchmark adult datasets.

The accuracy and purity of the original and perturbed datasets are compared and analysed using simulator tool Weka (Mark Hall et al 2009). Various classification algorithms like C4.5, Naïve Bayesian and Ripper and K-Nearest Neighborhood Classifier and EM clustering algorithm are taken for experimentation in this work. The Privacy preserved Adult and Medical Datasets versions are abbreviated as given in table 2 .

The utility of the privacy preserved datasets on classification data mining function is compared using accuracy, root mean square error and ROC-area metrics. The classification algorithms like Ripper, C4.5, K- Nearest Neighbourhood, Naive Bayesian are used for experimentation and the results are shown in the figures 7 to 12 .

The accuracies of the proposed privacy preserved adult datasets on the classification algorithms Ripper, C4.5, K- Nearest Neighbourhood, and Naive Bayesian are compared with the original adult dataset and adult L-diversified dataset and is shown in figure 7.

Table 2. Versions of datasets.

\begin{tabular}{lcc}
\hline S.No. & Privacy preserved datasets & Abbreviation \\
\hline 1 & Original Adult & OA \\
2 & Adult Entropy Euclidean K-means clustered dataset with cluster size C=2 & AEEKC2 \\
3 & Adult Entropy Manhattan K-means clustered dataset with cluster size C=2 & AEMKC2 \\
4 & Adult Information Gain Euclidean K-means clustered dataset with cluster size 2 & AIGEKC2 \\
5 & Adult Information Gain Manhattan K-means clustered dataset with cluster size C=2 & AIGMKC2 \\
6 & Adult L-Diversified & A-L-Diversity \\
7 & Original Medicine & OM \\
8 & Medical Entropy Euclidean K-Means Clustered dataset with cluster size C=2 & MEEKC2 \\
9 & Medical Entropy Manhattan K-Means Clustered dataset with cluster size C=2 & MEMKC2 \\
10 & Medical Information Gain Euclidean K-Means Clustered dataset with cluster size C=2 & MIGEKC2 \\
11 & Medical Information Gain Manhattan K-means clustered dataset with cluster size=2 & MIGMKC2 \\
12 & Medical L-Diversified & M-L-Diversity \\
\hline
\end{tabular}




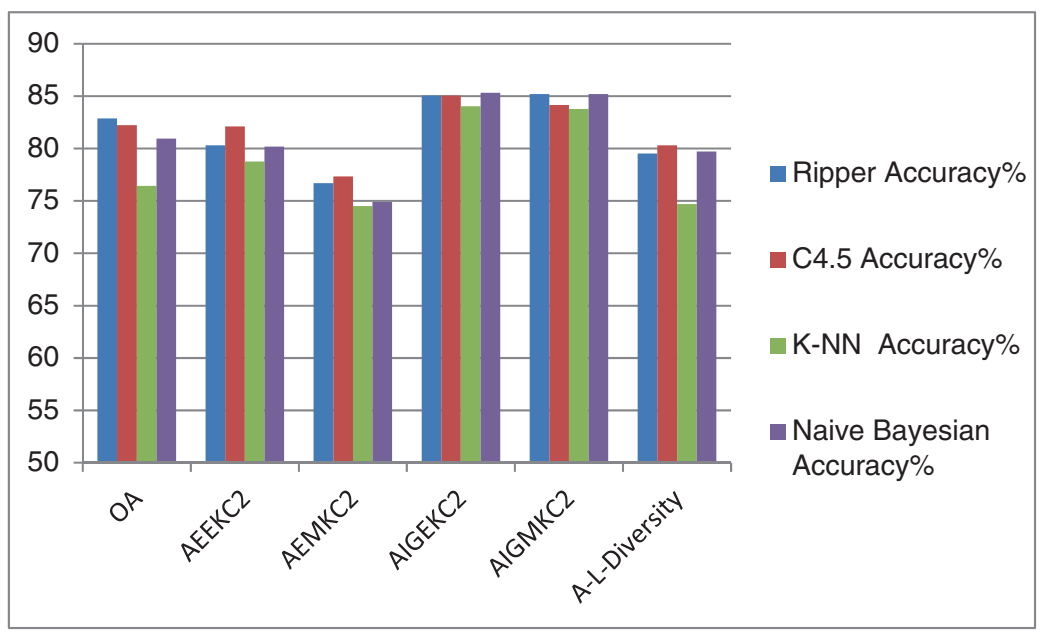

Figure 7. Accuracy comparisons of classification algorithms on privacy preserved adult datasets.

From figure 7 , it is inferred that the classification accuracies of dataset perturbed using proposed IGAPP method and EAPP method have comparable accuracy with original and Ldiversified datasets except for AEMKC2 dataset. Also, datasets perturbed using IGAPP method has more utility than the dataset perturbed using EAPP method on Adult dataset.

The accuracy of the privacy preserved medical dataset using the proposed methods on the classification algorithms taken for study is compared with the accuracy of the original medical dataset and L-diversified medical dataset and is shown in figure 8.

On medical dataset all the privacy preserved datasets using both IGAPP method and EAPP method have the same accuracy values as that of the original and L-diversified datasets on all the classification algorithms taken for experimentation.

Thus, experiments show that partitioning the dataset horizontally using distance namely, Manhattan or Euclidean and vertically partitioning the attributes using Entropy or Information Gain values have negligible effect on the classification accuracies. Thus, utility of the both adult and medical perturbed datasets are comparable with the original and L-diversified datasets.

To evaluate the utility of the privacy preserved adult datasets, the ROC area values of these datasets on the classification algorithms taken for study is compared with the original adult dataset and is shown in figure 9. Figure 9 shows that all the perturbed adult dataset versions except for EAPP perturbed datasets, have almost the same ROC value as that of the original adult dataset and L-diversified dataset.

The receiver operating characteristics (ROC) value of the privacy preserved medical datasets is compared with the original medical and L-diversified dataset and is shown in figure 10. Figure 10 shows that medical dataset versions perturbed using IGAPP method has a lower s ROC-value than the dataset versions perturbed using EAPP method. Thus, on real life medical dataset the vertical partitioning of attributes using information gain values leads to a decrease in its ROC values.

The RMSE values of the privacy preserved adult dataset on the classification algorithms taken for study is compared with the RMSE values of original and L-diversified adult dataset and is 


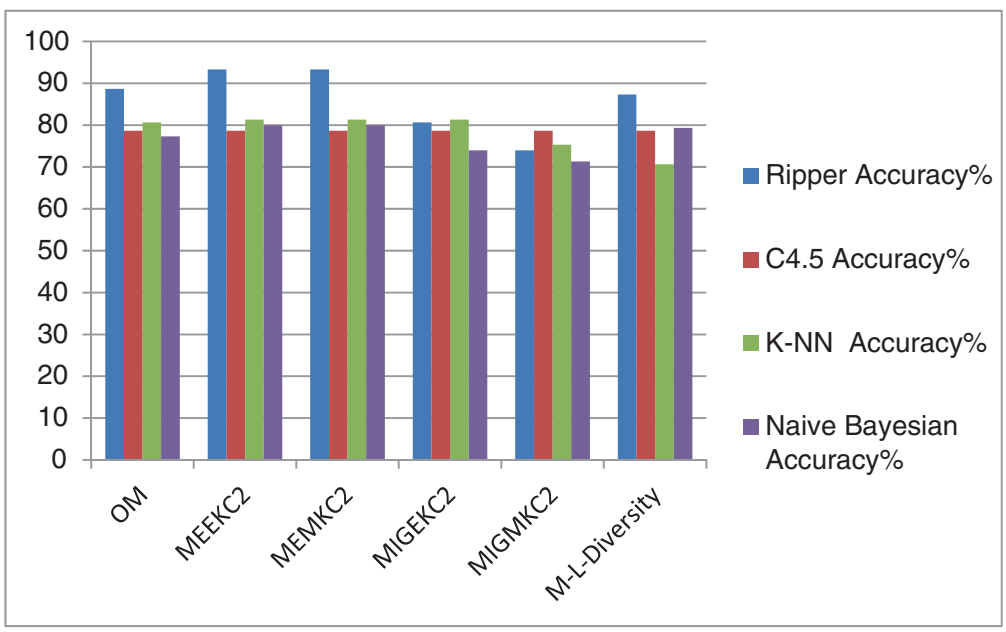

Figure 8. Accuracy comparisons of classification algorithms on privacy preserved medical datasets.

shown in figure 11. Figure 11 shows the comparison of RMSE values of the proposed privacy preserved versions of adult dataset with the original and L-diversified adult dataset. The graph indicates that AIGEKC2 and AIGMKC2 datasets have the least RMSE values and hence good utility than the other privacy preserved adult datasets.

The RMSE values of the privacy preserved medical dataset on the classification algorithms taken for study is compared with the RMSE values of the original medical dataset and is shown in figure 12. Figure 12 shows that RMSE values of datasets perturbed using EAPP method reveal a slightly higher RMSE values than the dataset perturbed using IGAPP method. Thus, the perturbed versions of adult dataset except for Manhattan entropy based perturbed dataset version have good utility values. On medical dataset, except for Manhattan IG based perturbed dataset, all other datasets have utility values better than the original and L-diversified dataset. This shows

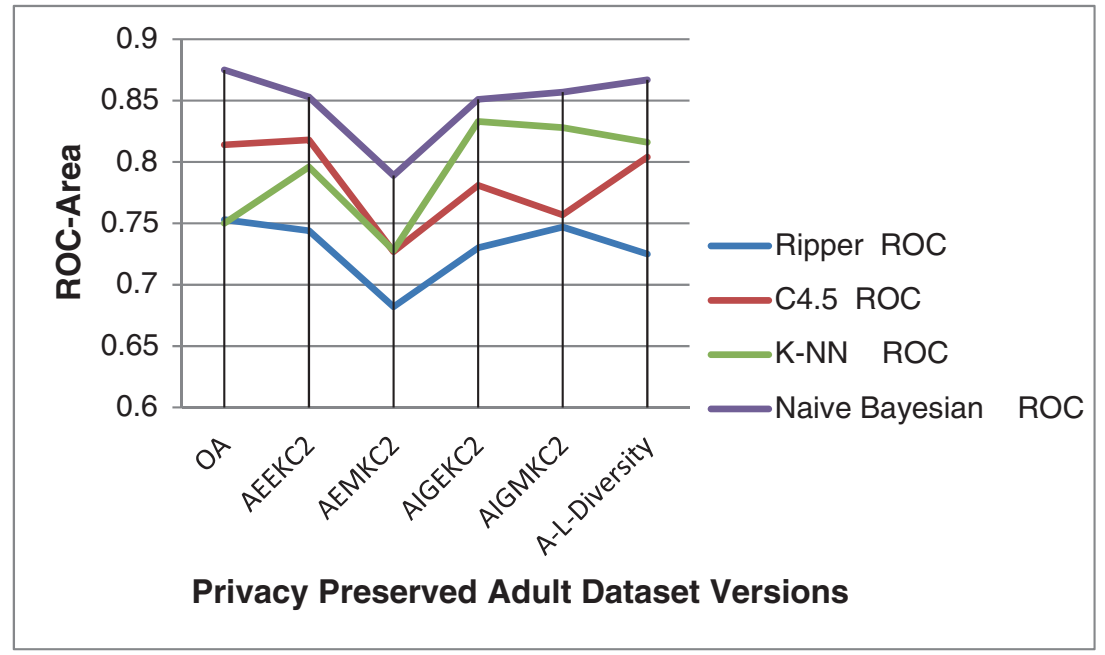

Figure 9. ROC-area values comparisons of the privacy preserved adult dataset. 


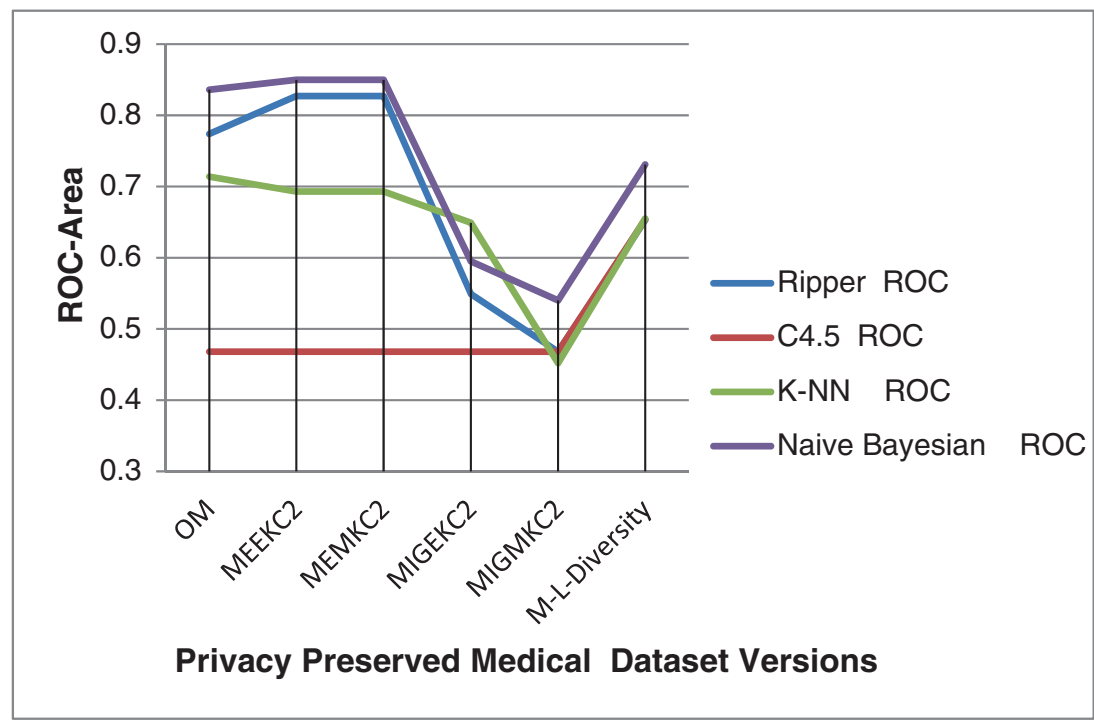

Figure 10. ROC-area values comparisons of the privacy preserved medical dataset.

that Manhattan distance based perturbed datasets have lower utility than Entropy distance based perturbed datasets.

Variance is a metric used in PPDM for measuring the degree in which the privacy preserved dataset varies from its original value. When the variance value of privacy preserved dataset is more, these versions cannot be merged with any other privacy preserved dataset versions to obtain the original dataset. This property helps in preventing diversity attack. The comparison of variance values of the privacy preserved versions of adult and medical datasets is shown in figures 13 and 14.

Figures 13 and 14 show that the dataset perturbed using IGAPP method have lesser variance values than the dataset perturbed using EAPP method. Thus, vertical partitioning of attributes

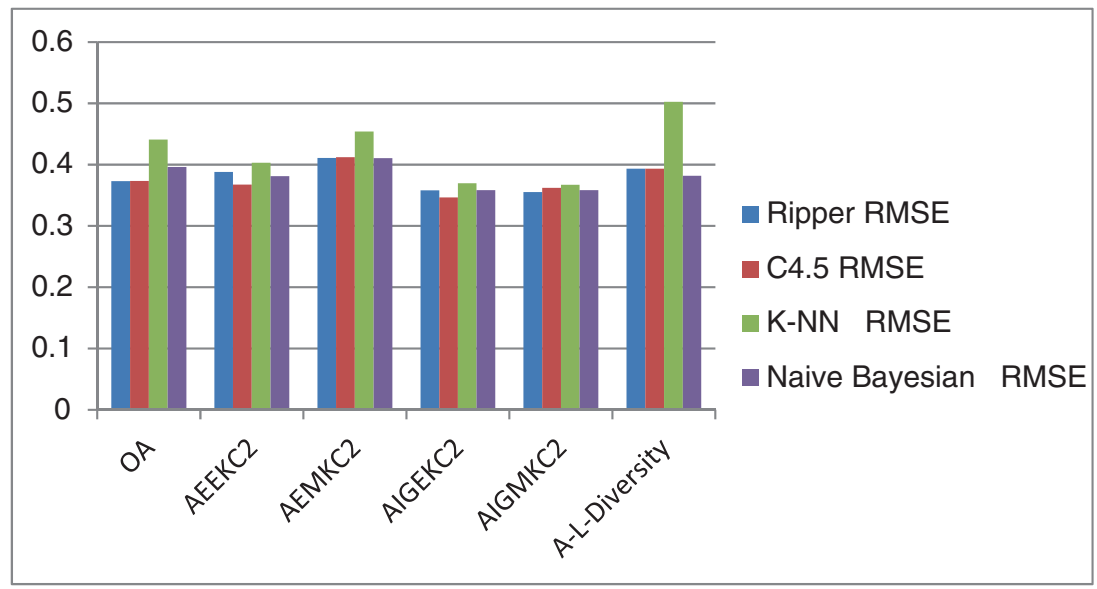

Figure 11. RMSE values comparisons of privacy preserved adult datasets. 


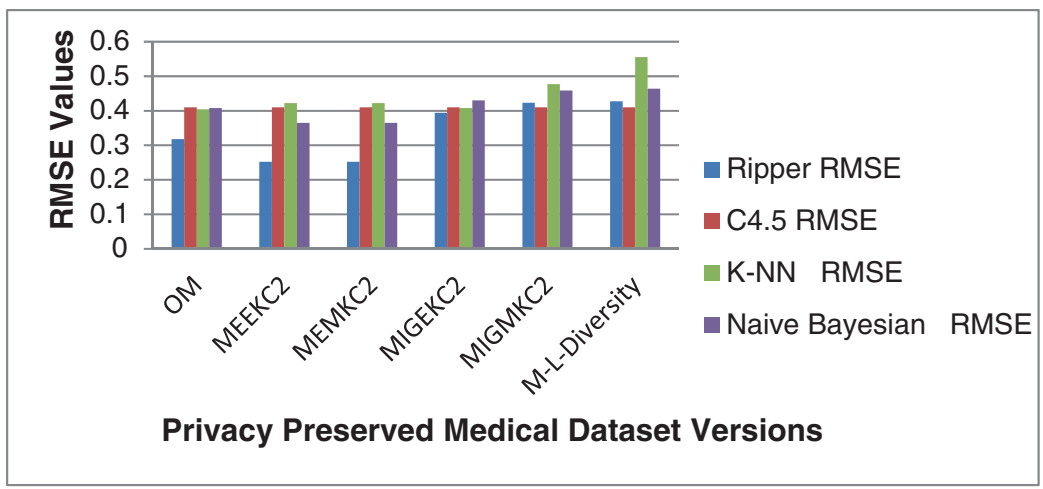

Figure 12. RMSE values comparisons of privacy preserved medical datasets.

using entropy value gives better variance than vertical partitioning of attributes using information gain value. Also, the graphs show that vertical partitioning has more effect on privacy preservation than horizontal partitioning.

Figures 15 and 16 compare the purity metric values of the perturbed dataset with that of the original dataset on EM clustering algorithm with the number of clusters $k=2,3$ and 4 . The purity of clustering results of perturbed adult datasets are compared with that of the original adult and L-diversified datasets on EM algorithm and are shown in figure 15.

The results show that the purity metric values of all the perturbed dataset using the proposed methods are higher than the original and L-diversified dataset. Also, adult dataset versions, AIGEKC2 and AIGMKC2 have higher purity values than the AEEKC2 and AEMKC2 datasets indicating that on adult dataset the proposed Information Gain based attribute perturbation method gives better clustering accuracy than in the proposed Entropy based attribute perturbation method.

The comparison of purity values on real life medical dataset using EM clustering algorithm is given in figure 16. Figure 16 shows that on real life medical dataset, the purity of the clustering result of perturbed datasets are lower than that of the original dataset when the number of clusters is 2 . Also, the purity values increase as the cluster size increases to 3 and 4 . In medical dataset, the purity values of the proposed privacy preserved datasets are higher than L-diversified datasets.

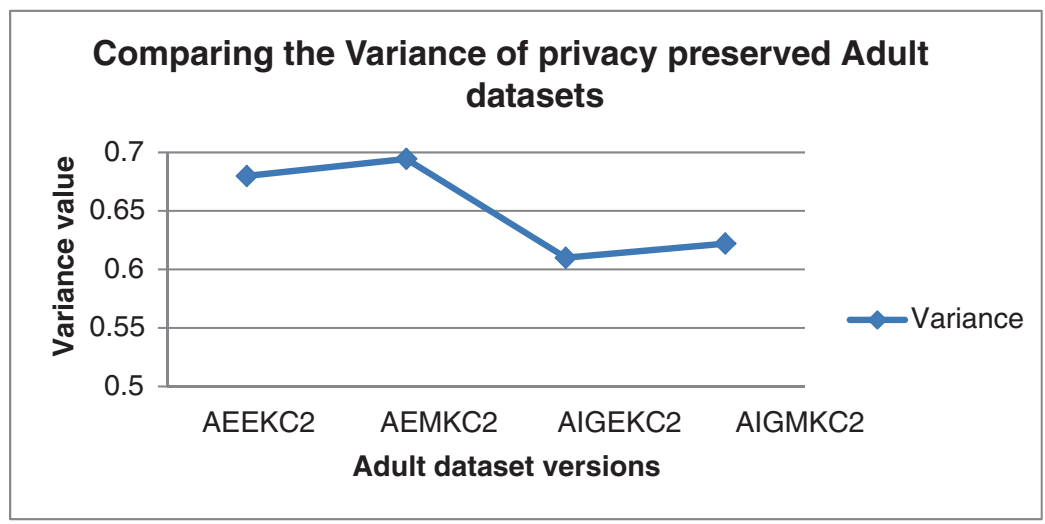

Figure 13. Variance values comparisons of privacy preserved adult datasets. 


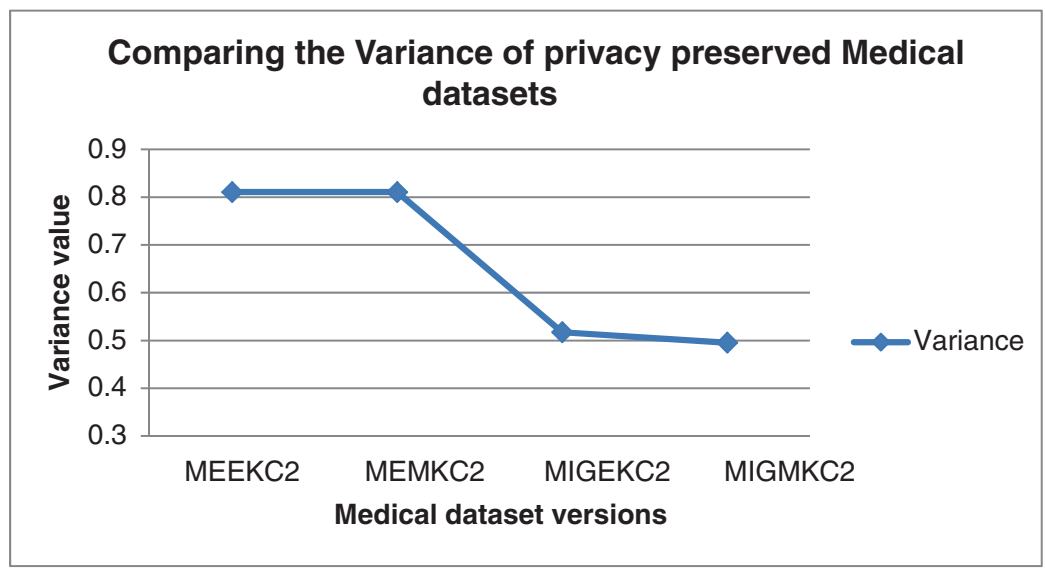

Figure 14. Variance values comparisons of privacy preserved medical datasets.

Thus, the datasets perturbed using the proposed methods has comparable utility on EM clustering algorithm as that of the original and L-diversified datasets.

Distortion metric measures the difference in the information gain rank values of the attributes in the dataset after privacy preservation. The levels of distortion of the privacy preserved datasets are measured using the metrics $\mathrm{CP}$ and $\mathrm{CK}$ respectively and tabulated (table 3 ).

$\mathrm{CP}$ values are directly proportional to the level of distortion in the datasets while $\mathrm{CK}$ value is inversely proportional to the level of distortion. It is inferred from table 3 that Manhattan distance based perturbed datasets of both adult and medical datasets using IGAPP and EAPP methods have the highest level of distortion of 3.27 and 2, respectively than the datasets perturbed using euclidean distance based perturbation methods. However, the distortion values of the proposed privacy preserved medical datasets are lower than that of the L-diversity perturbed dataset. This may be due to the fact that the medical datasets are subjected to preprocessing level generalization to hide sensitive values before being subjected to privacy preservation using the proposed methods.

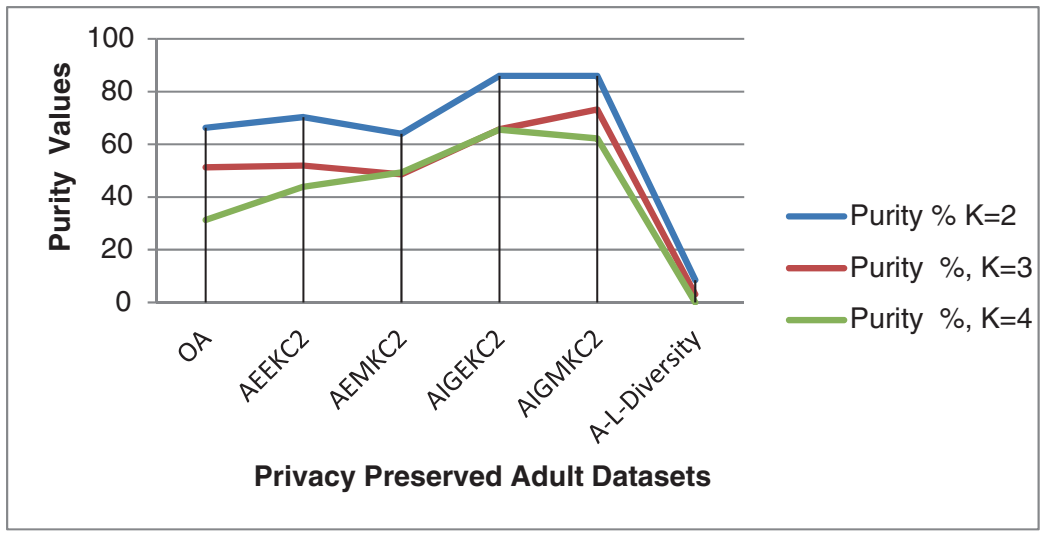

Figure 15. Purity value comparisons of EM clusters on perturbed adult dataset. 


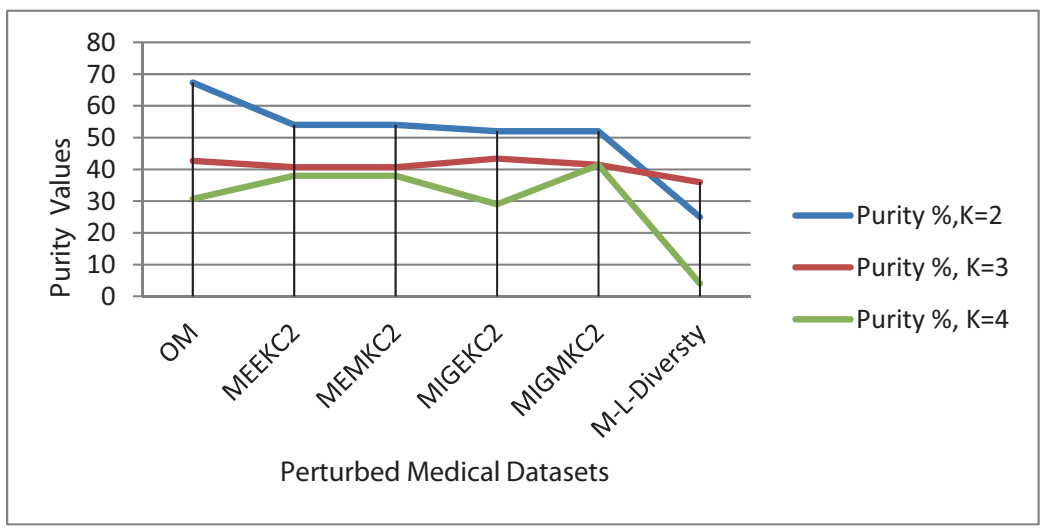

Figure 16. Purity values comparisons of EM clusters on perturbed medical dataset.

From the above results, it is inferred that the privacy preserved datasets have comparable utility on both clustering and classification functions of data mining than the original dataset and L-diversity perturbed dataset. Thus, four versions of the proposed privacy preserved datasets produced using the EAPP and IGAPP methods exhibit the following advantages:

- The preservation of nearest neighborhood structure of the data after masking which is evaluated using the accuracy of KNN algorithm and found to be comparable to the original dataset.

- In the multi-trust level environment the most common attack is diversity attack i.e., the individual masked versions cannot be joined to re-construct the original data. In the proposed frame work, perturbed dataset have different variance values thereby preventing the reconstruction of original data by combining the masked versions.

- As the datasets are subjected to two-dimensional partitioning, each attribute that lies within the cluster may be treated with two different perturbation techniques.

- When the IG values of all the proposed perturbed attributes are compared with the attributes of the original dataset and their distortion is measured using $\mathrm{CK}$ and $\mathrm{CP}$ metrics it is observed that datasets which are perturbed using IGAPP method are more distorted than

Table 3. CK and CP values comparisons of privacy preserved adult and medical datasets.

\begin{tabular}{cccc}
\hline S.No. & Privacy Preserved Datasets & CK-value & CP-value \\
\hline 1 & AEEKC2 & 0.36 & 1.45 \\
2 & AEMKC2 & 0.27 & 3.27 \\
3 & AIGEKC2 & 0.63 & 1.09 \\
4 & AIGMKC2 & 0.27 & 3.27 \\
5 & A-L-Diversity & 0.18 & 3 \\
6 & MEEKC2 & 0.166 & 2 \\
7 & MEMKC2 & 0.166 & 2 \\
8 & MIGEKC2 & 0.33 & 1.41 \\
9 & MIGMKC2 & 0.25 & 2.1 \\
10 & M-L-Diversity & 0.25 & 2.5 \\
\hline
\end{tabular}


the datasets perturbed using EAPP methods. However, these datasets have lesser variance with the original dataset.

- Also, it is observed that the IG values of perturbed attributes have decreased. Also, about $80 \%$ of the attributes in the perturbed dataset maintain their ranks after perturbation using both EAPP and IGAPP methods.

- As entropy value deals with the probability distribution among the attributes, perturbation based on these values will disturb the probability distribution and the associations between these attributes, thereby preventing the link among the attributes.

- Perturbation based on IG values will reduce the symmetry among the attributes thus preventing the privacy breach due to this correlation.

- Thus, values of CP and CK metrics of the attributes show that the one-way and two-way associations between attribute and class attributes have been distorted. Hence, the dependencies among these attributes will also be distorted.

\section{Conclusions}

In this work, datasets have been clustered using Simple K-means clustering algorithm with distance functions Euclidean and Manhattan with cluster size $\mathrm{K}=2$. The proposed IGAPP and EAPP method partitions data both vertically and horizontally on adult and medical datasets and applies privacy preservation techniques on them. The results show that horizontal partitioning based privacy preservation using euclidean distance have better utility than Manhattan distance. Also, the Manhattan distance perturbed datasets are more distorted than euclidean distance based perturbed datasets in both the datasets taken for experimentation. On privacy preservation variance metric, adult dataset versions have higher variance values than the real life medical dataset. This is due to the fact that in medical dataset the sensitive attributes and Quasi Identifiers have been subjected to first level of generalization as preprocessing, since they expose the sensitive information about the individuals in the dataset. The overall variance levels of all the perturbed datasets are above fifty percent from the original dataset and hence can prevent diversity attack. Thus, the proposed methods can be used to produce sequential release of datasets for multi trust level environment that can prevent diversity and linking attacks. The proposed two methods use information theory measures for automatic identification of associations among attributes and performs privacy preservation. This can be extended for privacy preservation in data streams since the characteristic of data streams have dynamic dimensionality which may expand and contract both vertically and horizontally. Hence, EAPP and IGAPP methods may be used for privacy preservation in data streams, as finding associations among attributes are automated.

\section{References}

Aggarwal C C and Philip S Y 2008 A general survey of privacy-preserving data mining models and algorithms, (pp. 11-52). Springer US

Baixeries Jaume 2008 A formal context for symmetric dependencies. Formal Concept Analysis, Berlin Heidelberg, Springer: 90-105

Benjamein Fung C M, Kewang, Lingyu Wang and Patrick Hang C K 2009 Privacy Persevering Data Publishing for Cluster Analysis. Data Knowl. Eng. 68: 552-575

Charu Aggarwal C and Philip Yu S 2008 An Introduction to Privacy Preserving Data Mining, Privacy Preserving Data Mining: Models and Algorithms. Springer Science, LLC 
Charu Aggarwal C 2008 Privacy and the Dimensionality Curse, Privacy Preserving Data Mining: Models and Algorithms. Springer Science, LLC

Chris R Giannela, Kunliu and Hillol Kargupta 2013 Breaching distance preserving data perturbation using few known inputs. Data Knowl. Eng. 83: 93-110

Danker F K and El Emam K 2012 The Application of Differential Privacy to Health Data, EDBT/ICDT Workshops, p 158-166

Dinusha Vatsalan, Peter Christen and Vassilios S Verykios 2013 A Taxonomy of Privacy Preserving Record Linking Techniques. Inf. Syst. 38: 946-969

Edoardo M Airoldi, XueBai and Bradley A Malin 2011 An Entropy Approach To Disclosure Risk Assessment: Lessons from Real Applications and Simulated Domains. Decis. Support Syst. 51: 1020

Erez shmueli, Tamir Tassa, Raz Wasserstein, Bracha Shapira and Loar Rokach 2012 Limiting disclosure of sensitive data on sequential releases of data bases. Inf. Sci. 191: 98-127

Evfimevski A, Gehrke J and Srikant R 2003 Limiting Privacy Breaches In: Privacy Preserving Data Mining, Proc. ACM PODS

Gagan Aggarwal, Tomas Feber, Krishnaram Kenthapadi, Samir Khuller, Rina Panigrahy, Dilys Tohas and Anzhu 2006 Achieving Anonymity via Clustering, PODS'06, June 26-28, Chicago

Grigorios Loukides, John Liagouris, Aris Gkoulalas-Divanis and Manolis Terrovitis 2014 Disassociation for electronic health record privacy. J. Biomed. Informatics 50: 46-61

Hall M A 1999 Correlation-based Feature Selection for Machine Learning (Doctoral dissertation, The University of Waikato). http://archive.ics.uci.edu/ml/datasets/Adult

Hui Wang and Ruilin Liu 2011 Privacy-preserving publishing microdata with full functional dependencies. Data Knowl. Eng. 70: 249-268

Ienco D, Pensa R G and Meo R 2012 From Context to Distance: Learning Dissimilarity for Categorical Data Clustering, ACM Trans. Knowl. Discovery Data 6(1)

Javier Herranz, Stan Matwin, Jordi Nin and Vicenc Torra 2010 Classifying data from protected statistical datasets. Comput. Security 29: 875-896

Jiawei Han, Micheline Kamber and Jian Pei 2011 Data Mining: Concepts and Techniques, 3rd Edition, Morgan Kaufmann

Josep Domingo - Ferrar 2008 A survey of inference control methods for privacy persevering data mining, Privacy Persevering Data Mining Models and Algorithms, Springer science + Business media, LLC

Lin Xiaodong, Clifton Chris and zhu Michael 2005 Privacy preserving clustering with distributed EM mixture modeling. Knowl. Inf. Syst. 8(1): 68-81

Li Liu, Murat Kantarcioglu and BhavaniThuraisingham 2008 The applicability of the perturbation based privacy preserving data mining for real-world data. Data Knowl. Eng. 65(1): 5-21

Mark Hall, Eibe Frank, Geoffrey Holmes, Bernhard Pfahringer, Peter Reutemann and Ian H Witten 2009 The WEKA Data Mining Software: An Update; SIGKDD Explorations, Volume, 11 (1)

Md Zahidul Islam and Lyilyana Brankovic 2011 Privacy Preserving Data Mining: A Noise Addition Framework Using a Novel Clustering Technique. Knowl. Based Syst. 24(8): 1214-1223

Ming Hau and Jian Pei 2008 A Survey Of Utility Based Privacy -Persevering Data Transformation Methods, Privacy Persevering Data Mining Models and Algorithms, Springer science + Business media, LLC

Murat Kantanlioglu 2008 A Survey Of Privacy Persevering Methods Across Horizontally Partitioned Data, Privacy Persevering Data Mining Models and Algorithms, Springer science + Business media, LLC

Oliveria S R M and Zaiana O R 2003 Privacy Preserving Clustering by Data Transformation, In: 18th Brezilian Symposium on Databases (SBBD 2003), 304-318

Pang-Ningtan, Michael Steinbach and Vipinkumar 2006 Introduction to data mining, Pearson Education, Inc

Rashid Hussain Khokhar, Rui Chen, Benjamin C M, Fung and Siu Man Lui 2014 Quantifying The Costs And Benefits of Privacy-Preserving Health Data Publishing. J. Biomed. Informatics 50: 107-121

Samarati P 2001 Protecting Respondents Identifies In Micro Data Release. IEEE Trans. Knowl. Data Eng. 13(6): 1010-1027 
Sarowar Sattar A H M, Jauyoug Li, Xiaofeng Ding, Jixue liu and Millist Vincent 2013 A general framework for privacy preserving data publishing. Knowl. Based Syst. 54: 276-287

Shlome Berkovsky, Tsvi Kufik and Francesco Ricci 2012 The Impact Of Obfuscation On the accuracy of collaborative filtering. Experts Syst. Appl. 39: 5033-5042

Songjie Gong 2011 Privacy Preserving Collabrative Filtering based Randomized Perturbation Techniques and Secure Multiparty Computing. Int. J. Adv. Comput. Technol. 3

Srujana Marugu and Joydeep Ghosh 2005 A privacy sensitive approach to distributed clustering. Pattern Recognit. Lett. 26: 399-410

Stanley R M olivera and Osmar R Zainne 2007 A Privacy Preserving Clustering Approach Towards Secure And Effective Data Analysis For Business Collaboration. Comput. Security 26: 81-93

Sweeney L 2002 Achieving k-anonymity privacy protection using generalization and suppression. Int. J. Uncertain. Fuz 10(6): 571-588

Tamas S Gal, Thomas C Tucker, Aryya Gangopadhyay and Zhiyuan Chen 2014 A data recipient centered de-identification method to retain statistical attributes. J. Biomed. Informatics 50: 32-45

Thomas M Cover and Joy A Thomas 1991 Elements of Information Theory, John Wiley \& Sons, Inc. Print ISBN 0-471-06259-6 Online ISBN 0-471-20061-1

Tiancheng Li, Ninghui Li, Jian Zhang and Ian Molloy 2012 Slicing: A New Approach for Privacy Preserving Data Publishing. IEEE Trans. Knowl. Data Eng. 24(3)

Wang J, Zhong W J, Zhang J and Xu S T 2006 Selective Data Distortion via Structural Partition and SSVD for Privacy Preservation, In: Proceedings of the 2006 International conference on Information and Knowledge Engineering, 114-120

Weijia Yang and Shang Tang Haung 2008 Data Privacy Protection In Multi Party Clustering. Data Knowl. Eng. 67: 185-199

Weiwei Ni and Zhihong Chong 2012 Clustering-Oriented Privacy Preserving Data Publishing. Knowl. Based Syst. 35: 264-270

Wu M and Zhang A D 2004 Feature Selection for Classifying High-Dimensional Numerical Data, Proceedings IEEE Computer Society Conference on Computer Vision and Pattern Recognition (CVPR2004), pp. 251-258

Yao Y 2003 Information-Theoretic Measures for Knowledge Discovery And Data Mining, In: Karmeshu, ed.: Entropy Measures, Maximum Entropy and Emerging Applications. Berlin Springer, 115-136

Yaping Li, Minghua Chen and Wei Zhang 2012 Enabling Multi-Level Trust in Privacy Preserving Data Mining. IEEE Trans. Knowl. Data Eng. 24(9): 1598-1612

Zhu D, Li X B and Wu S 2009 Identity disclosure protection: A data reconstruction approach for privacypreserving data mining. Decis. Support Syst. 48(1): 133-140 